\title{
Amphiphilic Gold Nanoparticles with V-shaped Arms
}

\author{
Eugene R. Zubarev, * Jun Xu, Arshad Sayyad, and Jacob D. Gibson \\ Department of Chemistry, Rice University, Houston, Texas 77005
}

\section{Supporting Information}

General. Unless otherwise stated, all starting materials were obtained from commercial suppliers and used without further purification. The ${ }^{1} \mathrm{H}$ NMR spectra were recorded on solutions in $\mathrm{CDCl}_{3}$ on a Varian Unity $300(300 \mathrm{MHz})$ spectrometer and Bruker $400 \mathrm{MHz}$ spectrometer. Size Exclusion Chromatography (SEC) analysis was conducted on a Waters Breeze 1515 series liquid chromatograph equipped with a dual $\lambda$ absorbance detector (Waters 2487), manual injector, and three styrogel columns (HR1, HR3, HR4) using linear polystyrene standards for calibration and tetrahydrofuran (THF) as the mobile phase. TEM images were obtained on a JEOL 1200EX scanning/transmission electron microscope operating at $100 \mathrm{kV}$ accelerating voltage and JEOL 2010 TEM microscope operating at $120 \mathrm{kV}$. Samples were prepared by casting one droplet of 0.1 wt. \% THF solution of mercaptophenol-functionalized Au NPs and $\mathrm{Au}(\mathrm{PB}-\mathrm{PEG})_{\mathrm{n}} \mathrm{NPs} \mathbf{1}$ onto carbon-coated copper TEM grids (continuous carbon film, Electron Microscopy Sciences) followed by drying for $2 \mathrm{~h}$. UV-vis spectra were collected on Shimadzu 2100 UV-VIS Spectrophotometer using 1 wt. \% THF solutions of Au NPs. Centrifugal filters (2 $\mathrm{mL}$ capacity) Ultrafree ${ }^{\circledR}$-CL containing regenerated cellulose membranes with molecular weight cut-off 30,000 g/mole were purchased from Fisher Scientific (cat.\# UFC4LTK25). Detailed synthesis of PB-PEG V-shaped amphiphile $2\left(\mathrm{M}_{\mathrm{w}}=3500 \mathrm{~g} / \mathrm{mole}\right.$ by MALDI-TOF, and $\mathrm{M}_{\mathrm{SEC}}=7120 \mathrm{~g} / \mathrm{mole}, \mathrm{M}_{\mathrm{w}} / \mathrm{M}_{\mathrm{n}}=1.1$ ) was described in our previous publication (see reference 12 in the text). 4-(N,N-dimethylamino)pyridinium-4-toluenesulfonate (DPTS) was prepared by mixing saturated THF solutions of $N, N$-dimethylaminopyridine (DMAP) (1 equiv) and $p$-toluenesulfonic 
acid monohydrate ( 1 equiv) at room temperature. The precipitate was filtered, washed several times with THF, filtered, and dried under vacuum. The structure of DPTS was confirmed by ${ }^{1} \mathrm{H}$ NMR.

Synthesis of mercaptophenol-functionalized gold nanoparticles. $300 \mathrm{mg}$ of hydrogen tetrachloroaurate trihydrate and $230 \mathrm{mg}$ of 4-mercaptophenol were dissolved in a mixture of 150 $\mathrm{mL}$ of methanol and $3 \mathrm{~mL}$ of acetic acid. Upon vigorous stirring, $30 \mathrm{~mL}$ of ice-cold $0.4 \mathrm{M}$ aqueous solution of $\mathrm{NaBH}_{4}$ was injected via syringe within few seconds. The reaction mixture changed color immediately from yellow-greenish to dark brown. After stirring for 30 minutes, the volume of the reaction mixture was reduced to approximately $50 \mathrm{~mL}$ on rotary evaporator at $45{ }^{\circ} \mathrm{C}$. The residue was diluted with $200 \mathrm{~mL}$ of $\mathrm{THF}$ and further with $400 \mathrm{~mL}$ of methylene chloride. Six extractions with pure water were carried out in order to remove the residual acetic acid, water, acetates, borohydrates, and methanol. The organic layer remained dark brown (almost black), whereas aqueous layers remained almost colorless. The organic layer was then evaporated to dryness on rotary evaporator at $45{ }^{\circ} \mathrm{C} .100 \mathrm{~mL}$ of methylene chloride were added and the dark brown material was sonicated for 30 seconds. After that $100 \mathrm{~mL}$ of hexane was added to cause partial precipitation of Au NPs (note that residual mercaptophenol is soluble in 50/50 mixture of hexane and methylene chloride). The resulting suspension was split into 6 vials and centrifuged for 30 minutes at $5000 \mathrm{rpm}$. The particles formed a thin film on the walls of the vials and the solutions became nearly colorless (slightly yellowish-brown). The solution containing residual mercaptophenol was removed by decantation. A new portion of methylene chloride was added to the precipitate followed by brief sonication and 15 min centrifugation. This was repeated 2 times until the solution showed no trace of UV-active mercaptophenol (a spot was placed onto TLC plate and checked under UV lamp with $254 \mathrm{~nm}$ wavelength). Finally, 
the particles were dissolved in $100 \mathrm{~mL}$ of THF and kept as a stock solution. The total weight of the product is typically $120-150 \mathrm{mg}$.

Synthesis of Au(PB-PEG) $)_{\mathbf{n}}$ NPs 1. A stock THF solution containing $7 \mathrm{mg}$ of $\mathrm{Au}(\mathrm{OH})_{\mathrm{n}} \mathrm{NPs}$ was evaporated on rotary evaporator at $45{ }^{\circ} \mathrm{C}$. Then $4 \mathrm{~mL}$ of $\mathrm{CH}_{2} \mathrm{Cl}_{2}$ and $50 \mathrm{mg}$ of PB-PEG amphiphile 2 were added into the flask. After complete dissolution of 2, $20 \mathrm{mg}$ of DPTS were added to the mixture. Two minutes later $0.15 \mathrm{~mL}$ of 1,3-diisopropylcarbodiimide (DIPC) were introduced dropwise upon vigorous stirring. Initially Au NPs do not dissolve and the activation of the carboxyl group of 2 takes place (conversion to anhydride) as confirmed by SEC. 5 minutes after addition of DIPC $1 \mathrm{~mL}$ of DMF was added in order to accelerate the dissolution of nanoparticles. Please note that even without DMF the particles will gradually dissolve and form dark brown solution (within several hours). The reaction can be easily monitored by TLC using $12 \% \mathrm{MeOH}$ in $\mathrm{CH}_{2} \mathrm{Cl}_{2}$. The new product forms within several minutes and its motion on TLC plate can be visualized without UV-lamp. The product has a dark brown color and its $\mathrm{R}_{\mathrm{f}}$ is very similar to that of the amphiphile $2\left(\sim 0.6\right.$ in $12 \mathrm{MeOH}$ in $\left.\mathrm{CH}_{2} \mathrm{Cl}_{2}\right)$. The $\mathrm{R}_{\mathrm{f}}$ of the starting material $\mathrm{Au}(\mathrm{OH})_{\mathrm{n}}$ is zero in this same mixture. The reaction typically takes $1-3 \mathrm{~h}$ as confirmed by TLC (nearly complete disappearance of $\mathrm{R}_{\mathrm{f}}=0 \mathrm{spot}$ ) and $\mathrm{SEC}$ (no change in intensity ratio between the peaks of $\mathbf{2}$ and 1). At this point $3 \mathrm{~mL}$ of DMF are added to the reaction mixture and nitrogen is gently blown above the solution in order to evaporate most of the methylene chloride. The resulting DMF solution is then split into three centrifugal filters (2 mL capacity) which are centrifuged at $5000 \mathrm{rpm}$. Within approximately $30 \mathrm{~min}$ the volume of the solution above the membrane of the filter is reduced to $0.2-0.3 \mathrm{~mL}$. The colorless solution containing all the low molar mass components, including the amphiphile $\mathbf{2}$, penetrates through membrane and is collected on the bottom of the filter. The dark brown solution concentrated on top of the 
membrane contains mostly the high molar mass product 1. New portion of DMF is then added into each filter bringing the volume back to $\sim 2 \mathrm{~mL}$ and the centrifugation is repeated for another 2 or 3 times until SEC shows no trace of the amphiphile $\mathbf{2}$ and its anhydride (double molecular weight). The DMF solution is then mixed with $5 \mathrm{~mL}$ of $\mathrm{CH}_{2} \mathrm{Cl}_{2}$ and 5-6 water extractions are used to remove DMF. The resulting solution is then evaporated giving $37 \mathrm{mg}$ of the pure product 1. ${ }^{1} \mathrm{H}$ NMR (300 MHz, $\mathrm{CD}_{2} \mathrm{Cl}_{2}$ ): $\delta 0.87$ (br, $6 \mathrm{H}, \mathrm{CH}_{3}$ of sec-Bu of $\mathrm{PB}$ ), 1.55-1.25 (br, 11H, $\mathrm{CH}_{2}$ of PB 1,2-addition units), 2.2-1.8 (br, 74H, aliphatic $\mathrm{CH}_{2}$ (both cis- and trans-) of 1,4addition units), 3.34 (s, 3H, terminal $\mathrm{CH}_{3}$ of PEO), 3.7-3.5 (br, 194H, $\mathrm{CH}_{2}$ from $\mathrm{PEO}$ ), 3.84 (br, 2H, PEO-CH $2-\mathrm{CH}_{2}-\mathrm{O}-$ ), 4.33 (br, 2H, PB- $\mathrm{CH}_{2}-\mathrm{CH}_{2}-\mathrm{O}-$ ), 4.50 (br, 2H, PEO-CH${ }_{2}-\mathrm{CH}_{2}-\mathrm{O}-$ ), 5.14.9 (br, 6H, vinyl $-\mathrm{CH}=\mathrm{CH}_{2}$ of $\mathrm{PB}$ 1,2-addition units), 5.5-5.3 (br, 30H, both cis- and trans$\mathrm{CH}=\mathrm{CH}$ of $\mathrm{PB}$ 1,4-addition units), 5.7-5.5 (br, 3H, vinyl $-\mathrm{CH}=\mathrm{CH}_{2}$ of $\mathrm{PB}$ 1,2-addition units), 8.3-7.3 (br, 21H, ArH, 16 protons from biphenyls, 3 protons from DHBA, and 4 protons from mercaptophenol). GPC (254 nm, THF), $\mathrm{M}_{\mathrm{w}}=40,334, \mathrm{PDI}=1.068$.

Estimation of the number of V-shaped molecules per Au NP. The average number of molecules 2 attached to Au NPs was estimated as follows. According to TEM (see Figure S3) the average diameter of the particles is $2 \pm 0.5 \mathrm{~nm}$. Assuming the truncated octahedral shape, a 2 nm cluster contains $\sim 270$ atoms of gold $\quad\left(\mathrm{N}_{\mathrm{Au} \text { atoms }}=4 \pi(\mathrm{d} / 2) / \mathrm{V}_{\mathrm{Au} \text { atom, }}\right.$, see reference $1 \mathrm{~S}$ and $2 \mathrm{~S})$. Therefore, the average molecular weight of Au NPs is $270 \times 197=53190 \mathrm{~g} / \mathrm{mole}$ and $7 \mathrm{mg}$ of $\mathrm{Au}$ NPs used in this synthesis contain $0.007 / 53190=1.316 \cdot 10^{-7}$ mole. This allows us to estimate the total number of $\mathrm{Au}$ particles reacted with the polymer $2 . \mathrm{N}_{\mathrm{AuNPs}}=1.316 \cdot 10^{-7} \times 6.023$. $10^{23}=7.926 \cdot 10^{16}$ particles. The number of molecules $2\left(\mathrm{M}_{\mathrm{w}}=3500\right.$ by MALDI $)$ in $30 \mathrm{mg}$ which 
were attached to the particles $(37-7=30 \mathrm{mg})$ is $\mathrm{N}_{\text {amphiphile } 2}=(0.03 / 3500) \times 6.023 \cdot 10^{23}=5.163$. $10^{18}$ molecules. Thus, there are $5.163 \cdot 10^{18} / 7.926 \cdot 10^{16} \cong 65$ molecules per particle.

This allows us to estimate the grafting density of the V-shaped molecules. The size of the Au cluster is $2 \mathrm{~nm}$ and the length of mercaptophenol is $\sim 0.8 \mathrm{~nm}$. Therefore the radius is $1.8 \mathrm{~nm}$ (assuming a spherical shape). The surface area is then $4 \pi r^{2} \cong 44 \mathrm{~nm} 2$, and the grafting density is $65 / 44=1.47 \mathrm{~V}$-shaped molecules per $\mathrm{nm}^{2}$. Each V-shaped has 2 chains (PB and PEG), which gives 2.94 chains $/ \mathrm{nm}^{2}$ as a grafting density. This is one of the highest grafting densities reported so far which demonstrates high efficiency of this synthetic approach. Other grafting densities reported in the literature include 0.3 chains $/ \mathrm{nm}^{2}$ for PMMA on Au NPs ("grafting from" method by living radical polymerization) as described in Reference 10d, and 2.0 chains/nm2 for $\mathrm{PEG}_{45}$ $(\mathrm{MW}=2000), 1.2$ chains $/ \mathrm{nm}^{2}$ for $\mathrm{PS}_{125}(\mathrm{MW}=13000)$, and 4.2 chains $/ \mathrm{nm}^{2}$ for PS19 $(\mathrm{MW}=2000)$ as described by Lennox in reference 10a.

The conversion of hydroxyl groups can also be estimated. As has been shown previously by Brust (ref. 13) and later confirmed by Astruc (JACS 2002, 124, 1782) the number of $n$ dodecanethiol chains on $2 \mathrm{~nm} \mathrm{Au} \mathrm{NPs} \mathrm{is} \mathrm{about} \mathrm{100.} \mathrm{If} \mathrm{we} \mathrm{assume} \mathrm{that} \mathrm{the} \mathrm{same} \mathrm{number} \mathrm{of}$ ligands is present when 4-mercaptophenol is used to passivate the surface of particles, then the conversion is $\sim 65 \%$. However, the cross-sectional area of 4-mercaptophenol is significantly larger than that of dodecanethiol and the number of these ligands is likely to be less than 100 which would make the conversion higher than $65 \%$. The accurate determination of the number of mercaptophenol ligands on $2 \mathrm{~nm}$ Au NPs is has not been done previously and represents a quite challenging task. There appears to be no data on that subject in the literature. 


\section{References}

1S. Brust, M.; Walker, M.; Bethell, D.; Schiffrin, D. J.; Whyman, R. J. Synthesis of Thiol-

Derivatized Gold Nanoparticles in a Two-phase Liquid-Liquid System. J. Chem. Soc., Chem.

Commun. 1994, 801-802.

2S. Labande, A.; Ruiz, J.; Astruc, D. Supramolecular Gold Nanoparticles for the Redox

Recognition of Oxoanions: Syntheses, Titrations, Stereoelectronic Effects, and Selectivity. J.

Am. Chem. Soc. 2002, 124, 1782-1789.

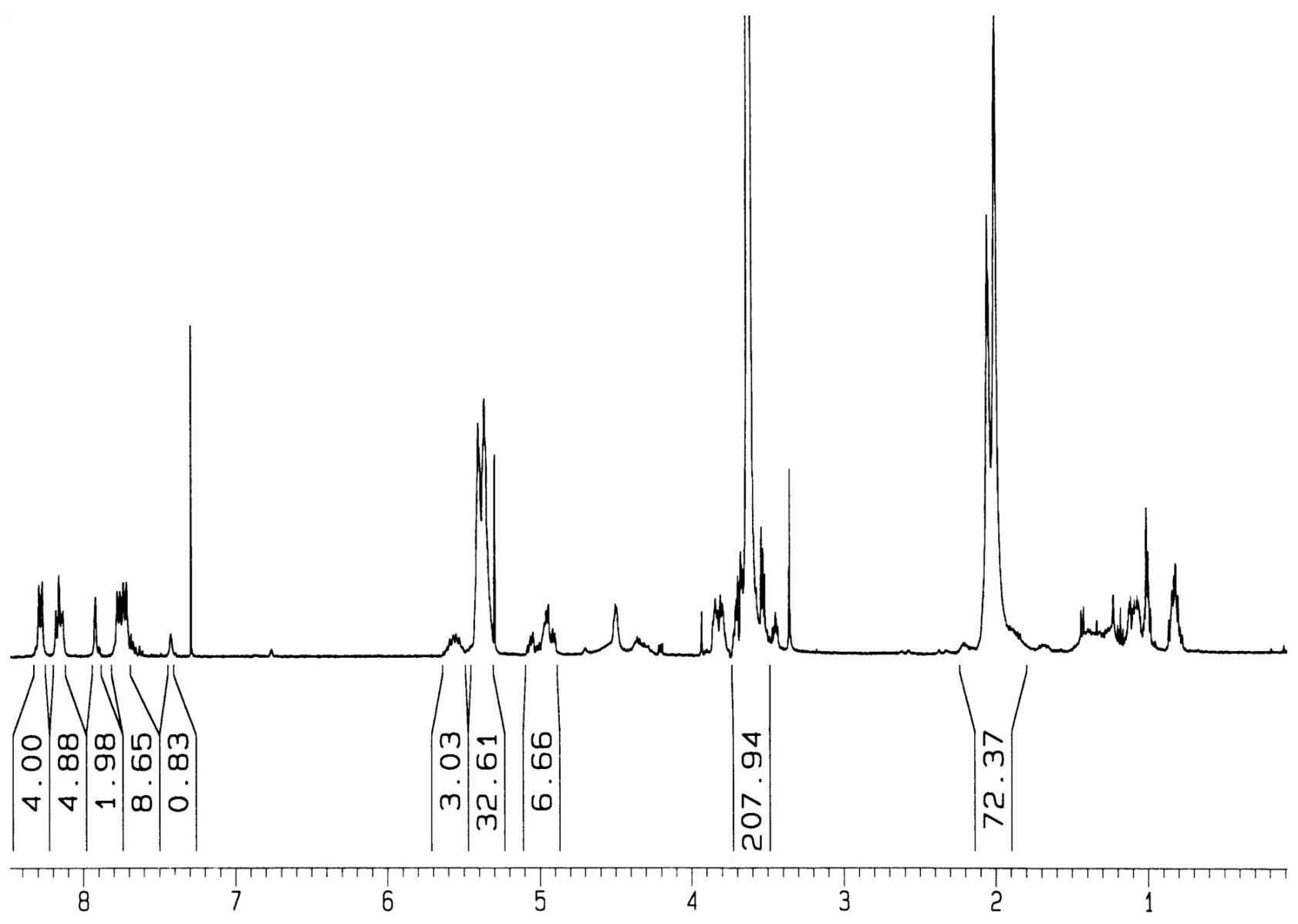

Figure S1. ${ }^{1} \mathrm{H}$ NMR of PB-PEG V-shaped amphiphile 2 in $\mathrm{CDCl}_{3}$. 


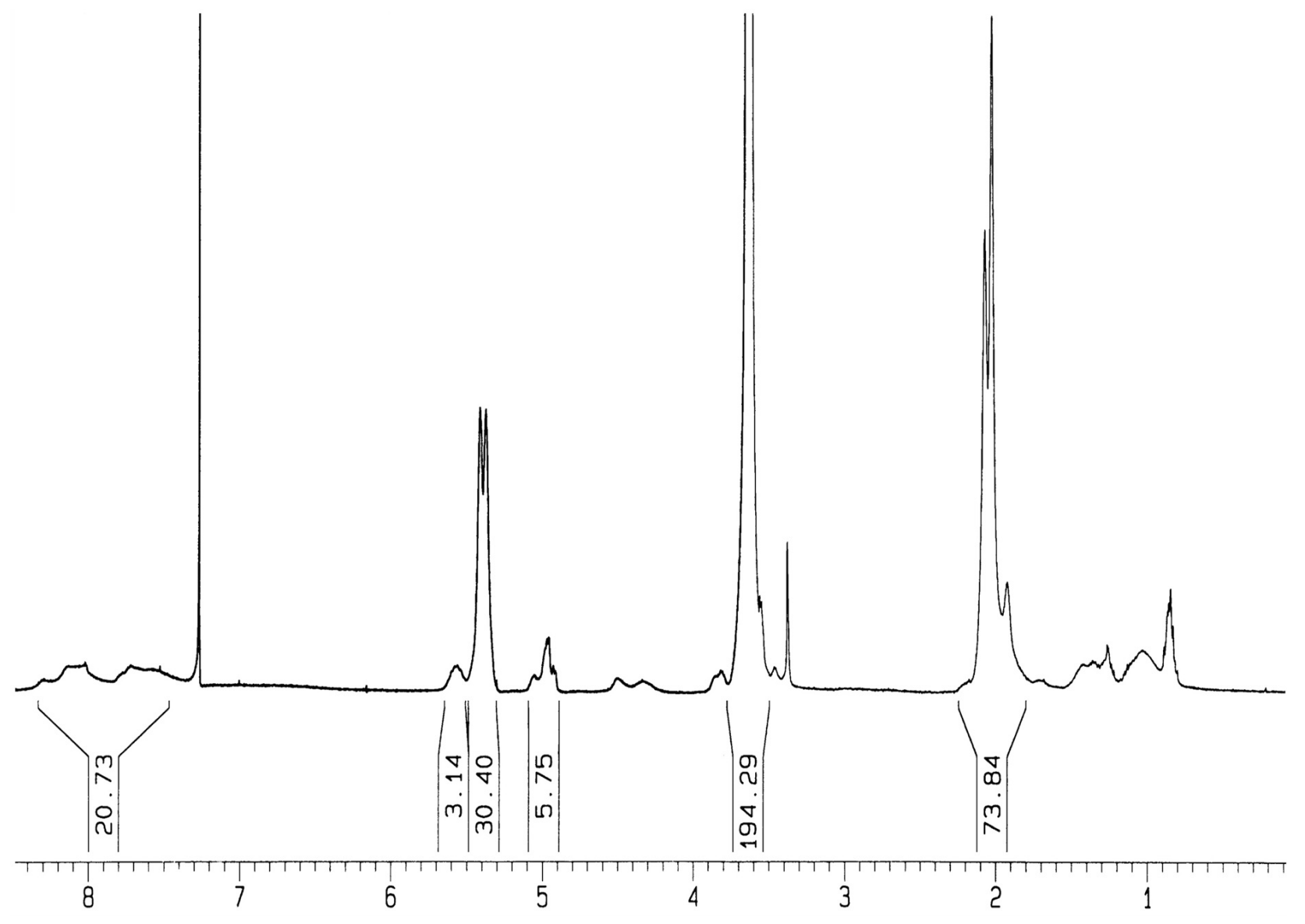

Figure S2. ${ }^{1} \mathrm{H}$ NMR of Au(PB-PEG $)_{\mathrm{n}}$ NPs 1 in $\mathrm{CDCl}_{3}$. Please note that the following sharp peaks from the spectrum of the amphiphile 2 (see Fig. S1) are not present: doublet at $8.32 \mathrm{ppm}$, triplet at $8.15 \mathrm{ppm}$, singlet at $7.88 \mathrm{ppm}$, doublet at $7.77 \mathrm{ppm}$, doublet at $7.72 \mathrm{ppm}$, siglet at $7.41 \mathrm{ppm}$. PB and PEG peaks also became much broader: 5.55, 5.0, 4.5, 4.3, 4.2, 3.8, 3.45, 3.31, 1.4-0.75. 


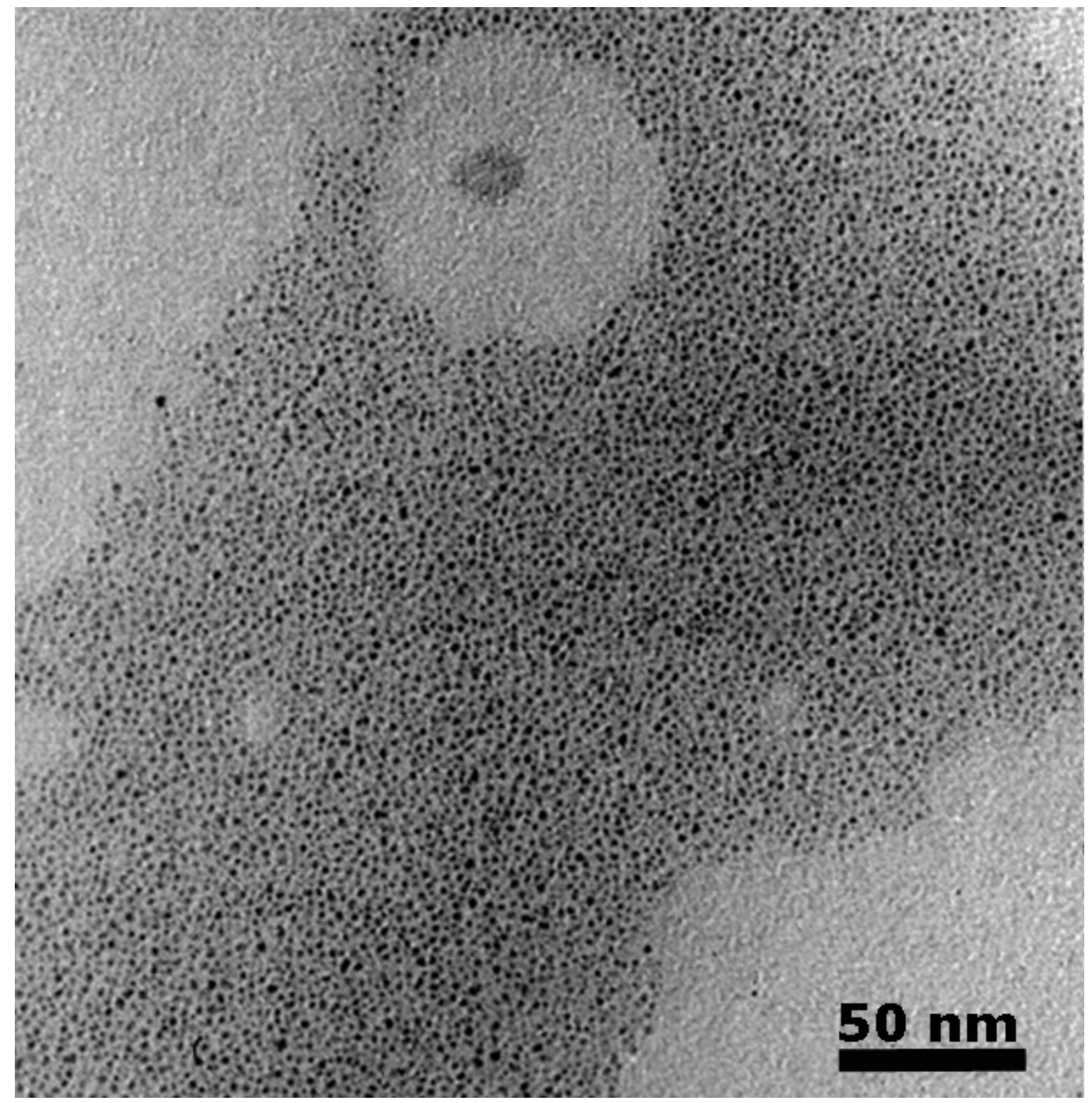

Figure S3. TEM image of mercaptophenol-functionalized Au NPs. 


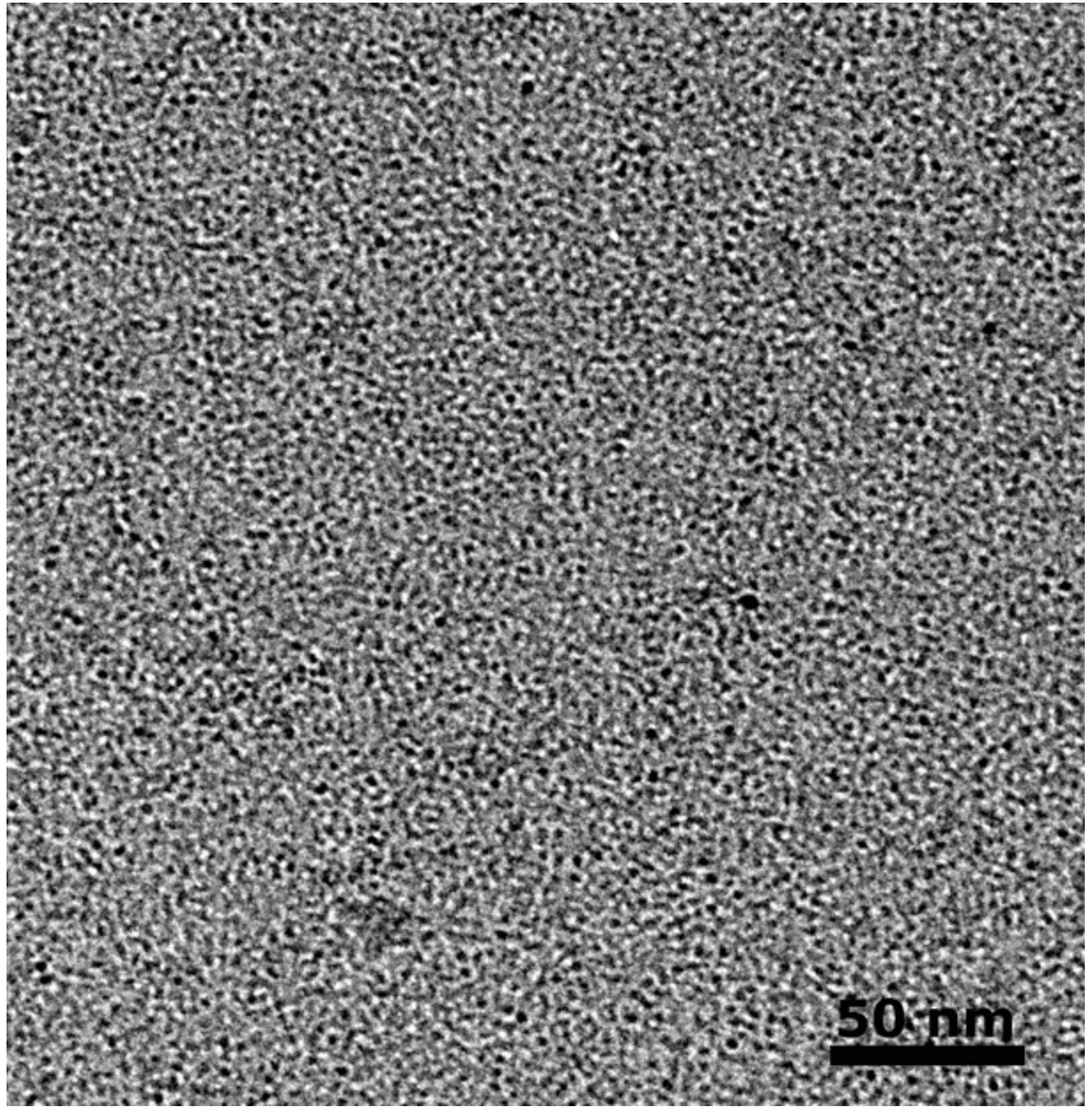

Figure S4. TEM image of Au(PB-PEG) $)_{\mathrm{n}}$ NPs 1 cast from 1 wt $\%$ chloroform solution. 


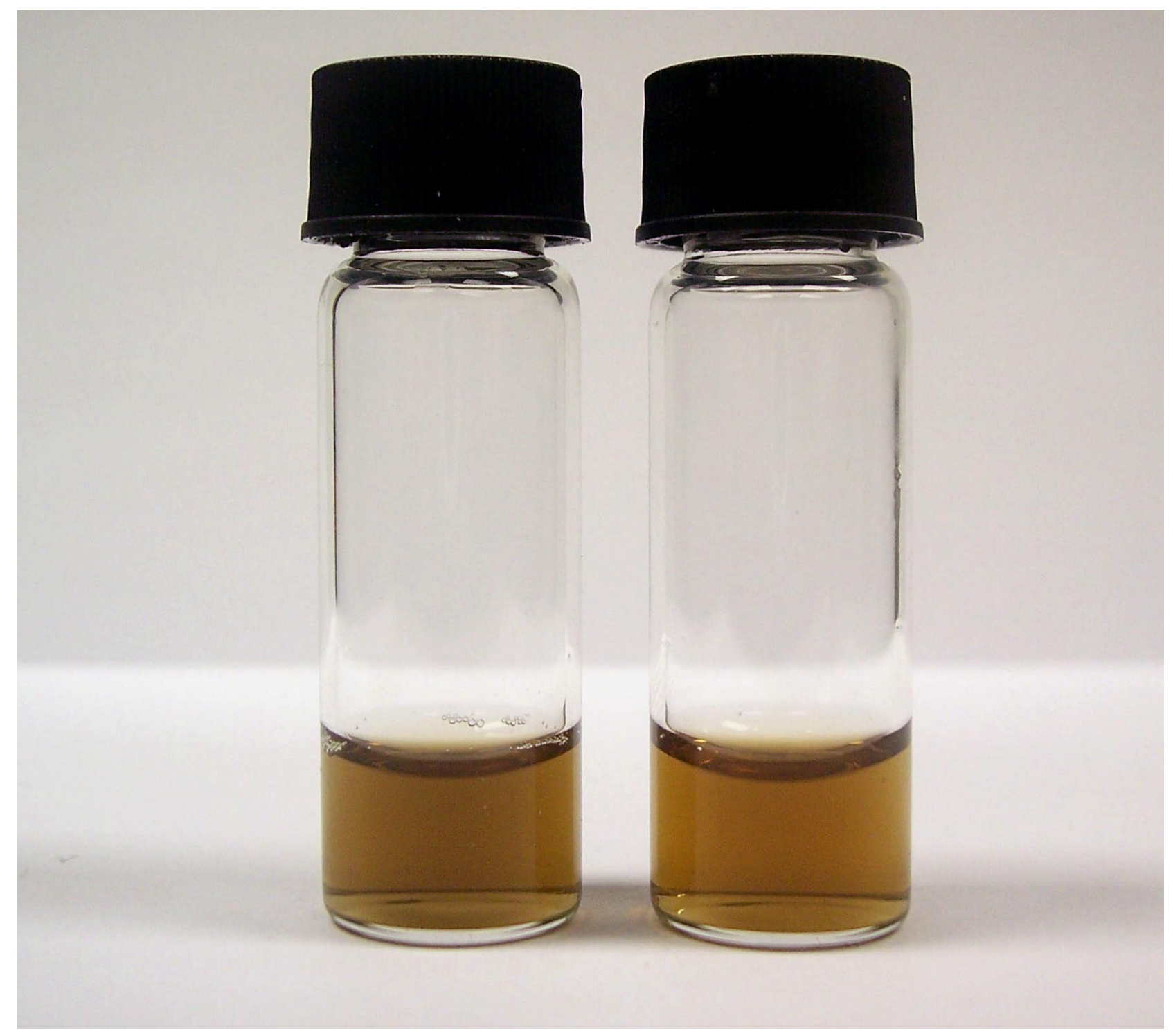

Figure S5. Photograph of freshly prepared (left) and 25-month-old (right) aqueous solution of $\mathrm{Au}(\mathrm{PB}-\mathrm{PEG})_{\mathrm{n}}$. 

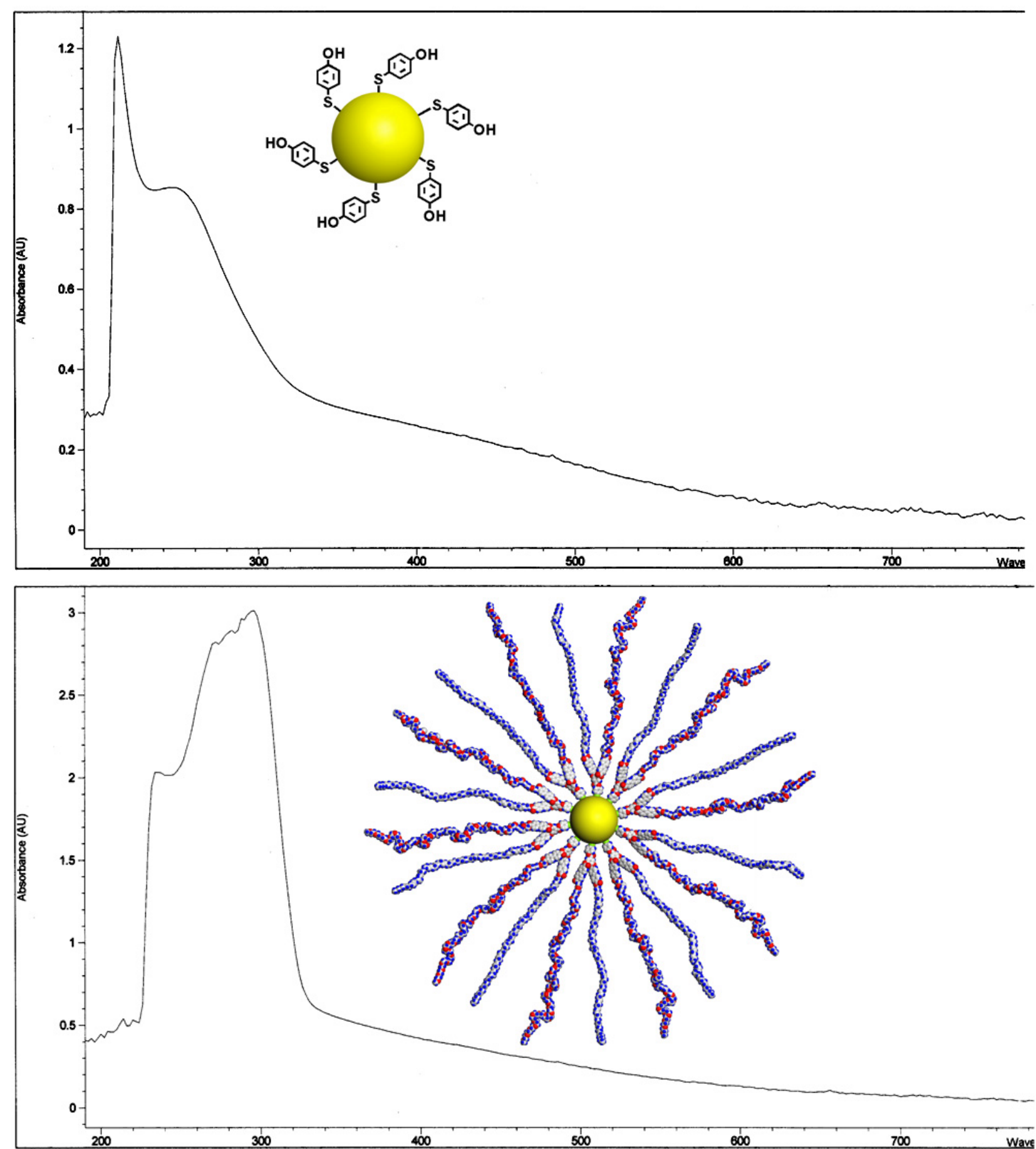

Figure S6. UV-vis spectra of mercaptophenol-functionalized Au NPs (top) and Au(PB-PEG) (bottom) collected from $1 \mathrm{wt}$ \% THF solutions. No plasmon peak can be detected around 520-530 $\mathrm{nm}$ which confirms that the size of Au clusters is below the critical size of $\sim 3 \mathrm{~nm}$. The absence of plasmon for small Au clusters (1-2 $\mathrm{nm}$ ) is the reason why they always retain their yellow-brown color regardless of concentration, the nature of solvent, and temperature. Even an aggregation of 2 $\mathrm{nm} \mathrm{Au}$ NPs does not change their color. Only when the size exceeds 3-3.5 nm the plasmon peak appears and the color of gold changes from brown to red. See reference 7 (Fig. 10) for example. 


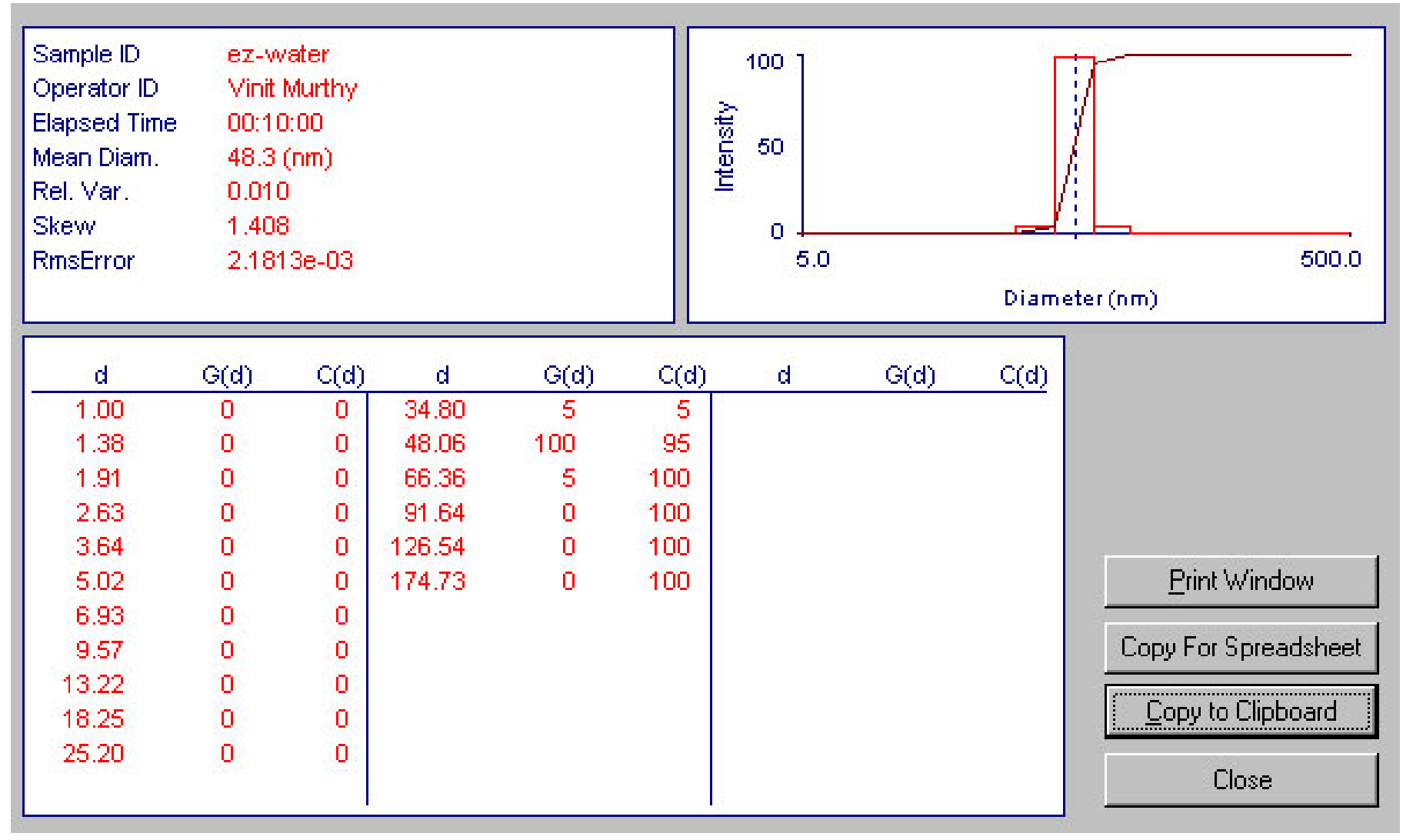

Figure S7. Dynamic light scattering result obtained from $0.3 \%$ solution of $A u(P B-P E O)_{n} 1$ in water. The mean diameter of the aggregates is $48.3 \mathrm{~nm}$. Because the size of the individual hybrid nanoparticles 1 is significantly smaller $(\sim 10 \mathrm{~nm})$ it is reasonable to assume that spherical micellelike aggregates exist in aqueous solution. However, the confirmation by other techniques is necessary before any definitive conclusion can be made.

Size distribution analysis was carried out with Brookhaven ZetaPALS dynamic light scattering (DLS) instrument with BI-9000AT digital autocorrelator at $656 \mathrm{~nm}$ wavelength. All studies were done at a $90^{\circ}$ scattering angle and temperature controlled at $25{ }^{\circ} \mathrm{C}$ in standard $4 \mathrm{ml}$ cuvettes. Measurements were made using "9KDLSW" software package and the results were averaged over a 10 min time period. 


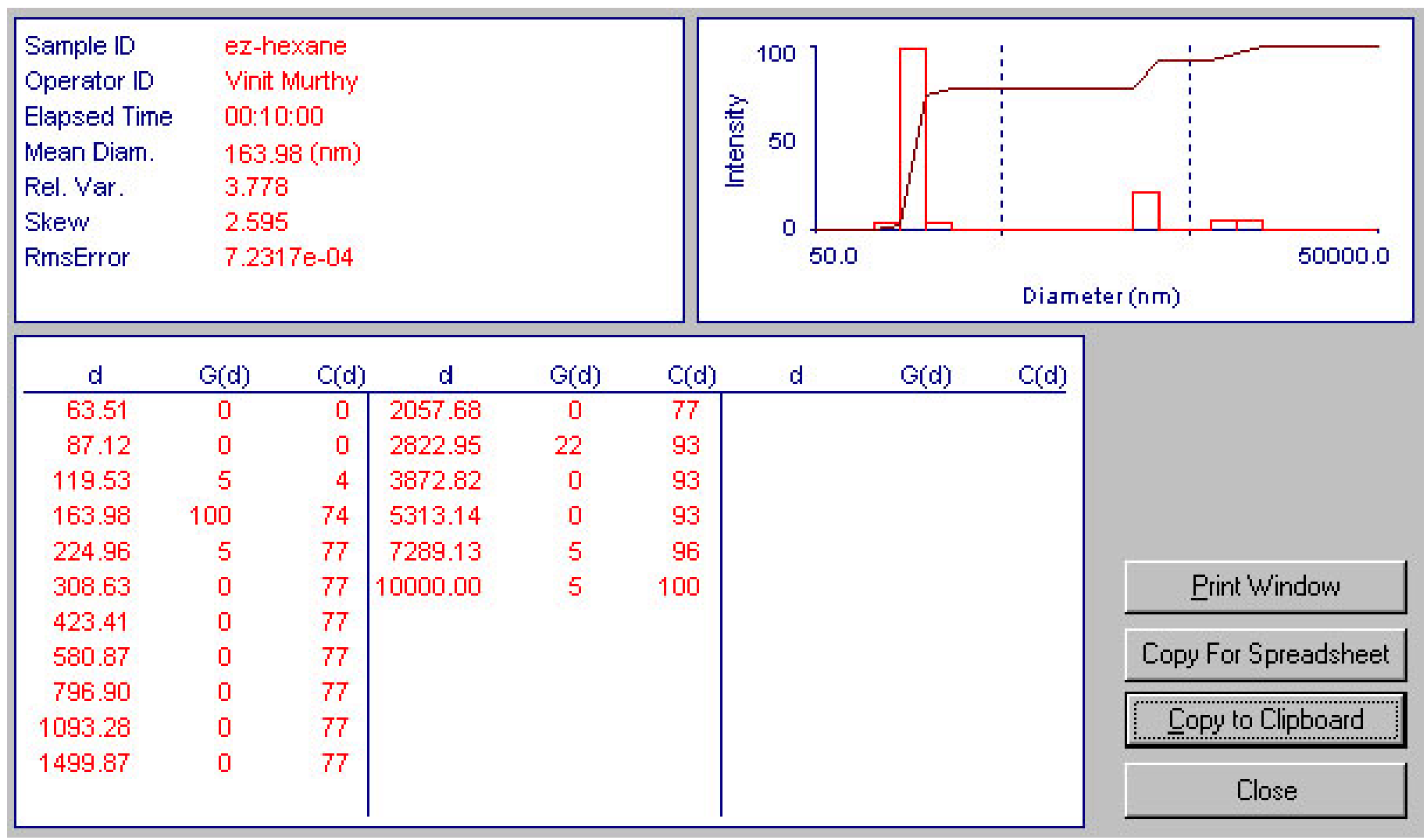

Figure S8. Dynamic light scattering result obtained from $0.3 \%$ solution of $\mathrm{Au}(\mathrm{PB}-\mathrm{PEO})_{\mathrm{n}} \mathbf{1}$ in hexane. The mean diameter of the aggregates is $163.98 \mathrm{~nm}$. Because the size of the individual hybrid nanoparticles $\mathbf{1}$ is significantly smaller $(\sim 10 \mathrm{~nm})$ it is reasonable to assume that some micelle-like aggregates (possibly vesicles) exist in hexane solution. However, the confirmation by other techniques is necessary before any definitive conclusion can be made.

Size distribution analysis was carried out with Brookhaven ZetaPALS dynamic light scattering (DLS) instrument with BI-9000AT digital autocorrelator at $656 \mathrm{~nm}$ wavelength. All studies were done at a $90^{\circ}$ scattering angle and temperature controlled at $25{ }^{\circ} \mathrm{C}$ in standard $4 \mathrm{ml}$ cuvettes. Measurements were made using "9KDLSW" software package and the results were averaged over a 10 min time period. 


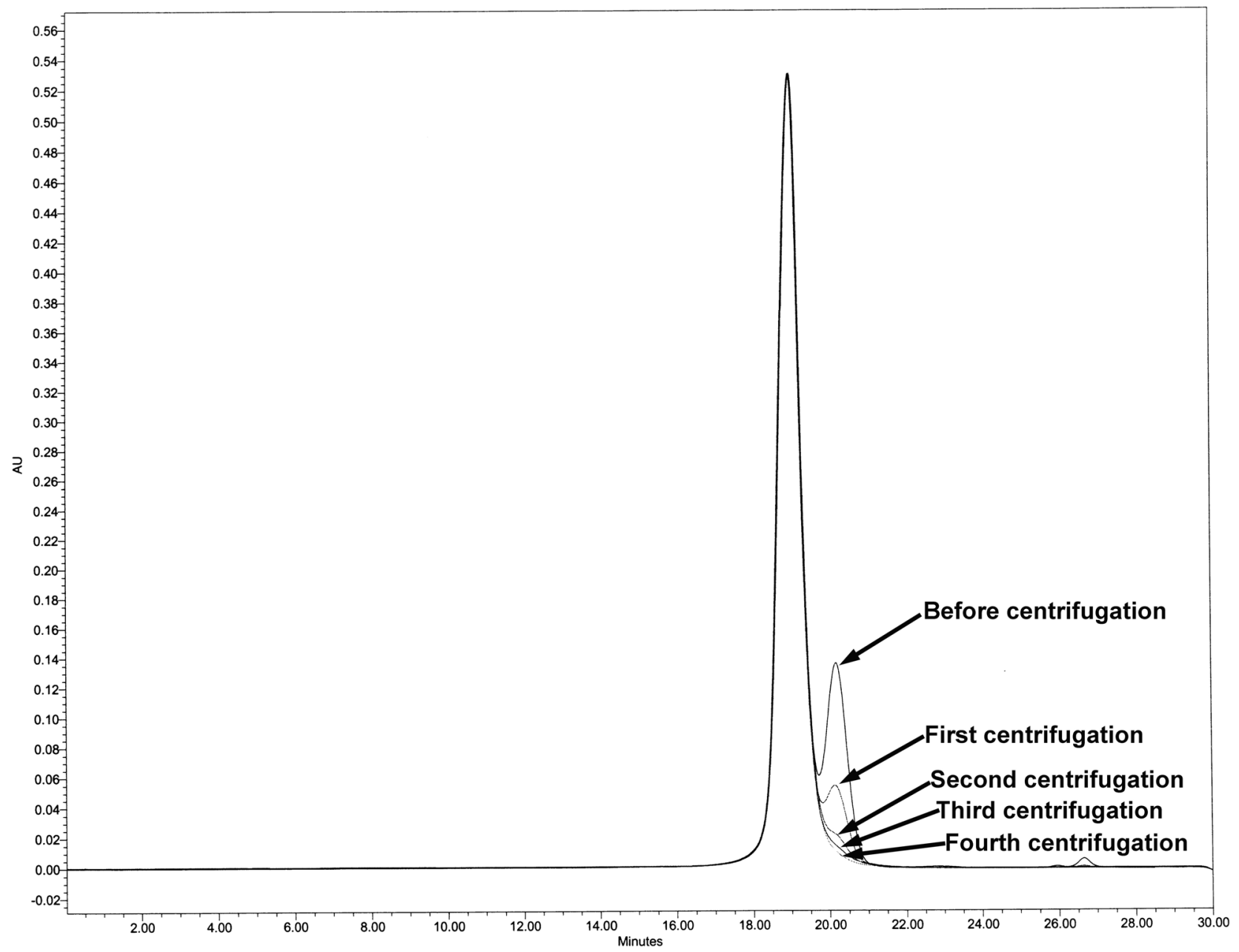

Figure S9. Overlapped GPC traces showing a decrease in the amount of V-shaped PB-PEO amphiphile 2 after four consecutive rounds of centrifugal ultrafiltration. The difference between the third and the fourth centrifugation is very small and the area under the tiny shoulder (tail) is less than $0.3 \%$ of the area under the main peak (high molar mass peak). This series of experiments suggests that the residual $\mathrm{V}$-shaped amphiphile $\mathbf{2}$ is almost completely removed. 

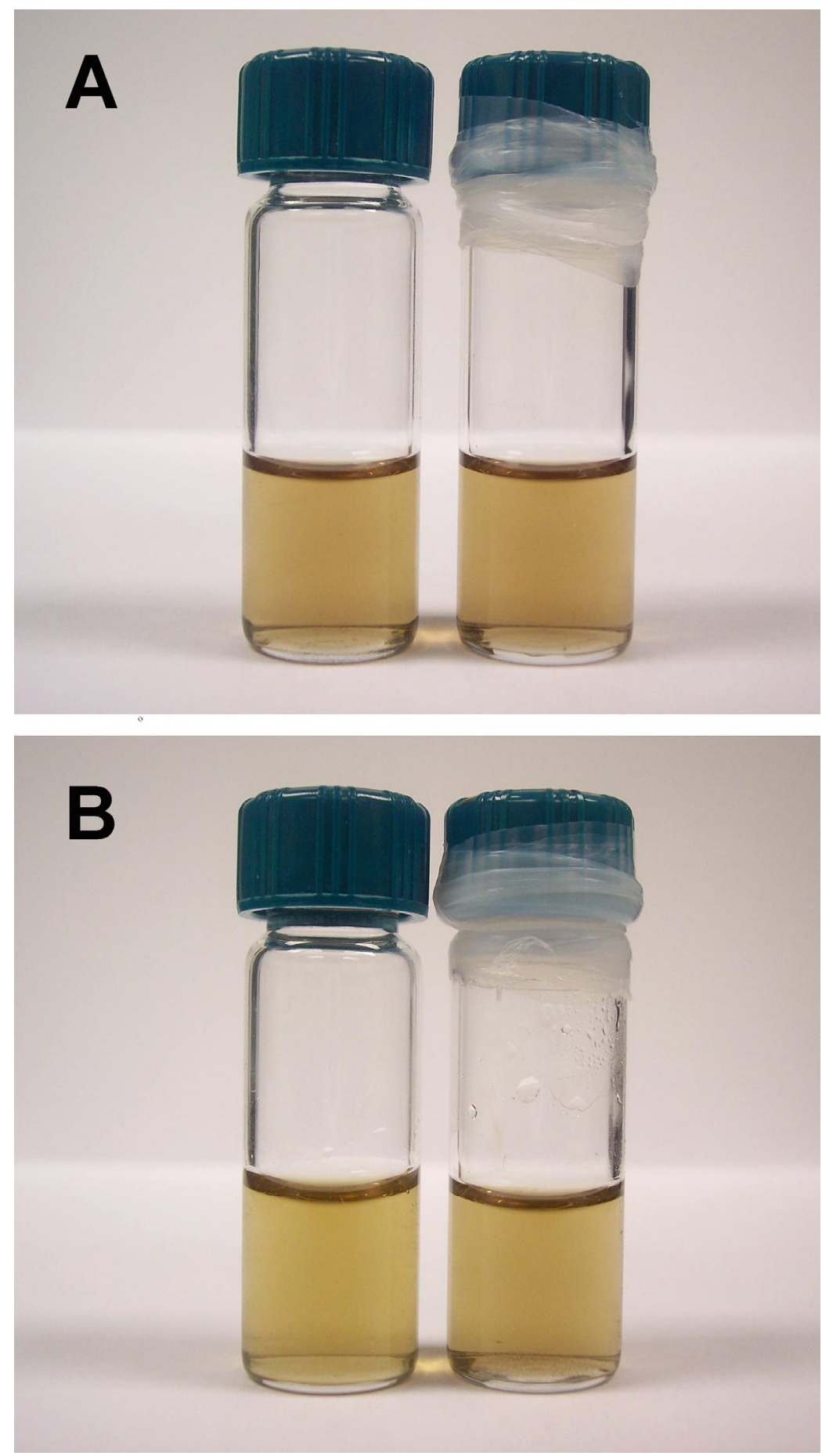

Figure S10. A: photographs of hexane solutions of $\mathrm{Au}(\mathrm{PB}-\mathrm{PEG})_{\mathrm{n}} \mathbf{1}$ before (left) and after annealing at $90{ }^{\circ} \mathrm{C}$ for 24 hours. B: photographs of water solutions of $\mathrm{Au}(\mathrm{PB}-\mathrm{PEG})_{\mathrm{n}} \mathbf{1}$ before (left) and after annealing at $90{ }^{\circ} \mathrm{C}$ for 24 hours. These experiments were carried out to determine the thermal stability of $\mathrm{Au}(\mathrm{PB}-\mathrm{PEG})_{\mathrm{n}} \mathbf{1}$ in selective solvents in which the aggregation takes place as confirmed by DLS experiments. Examination of the solutions by SEC after annealing showed that the high molecular weight peak remained at the same position and the polydispersity index did not change (1.07). 

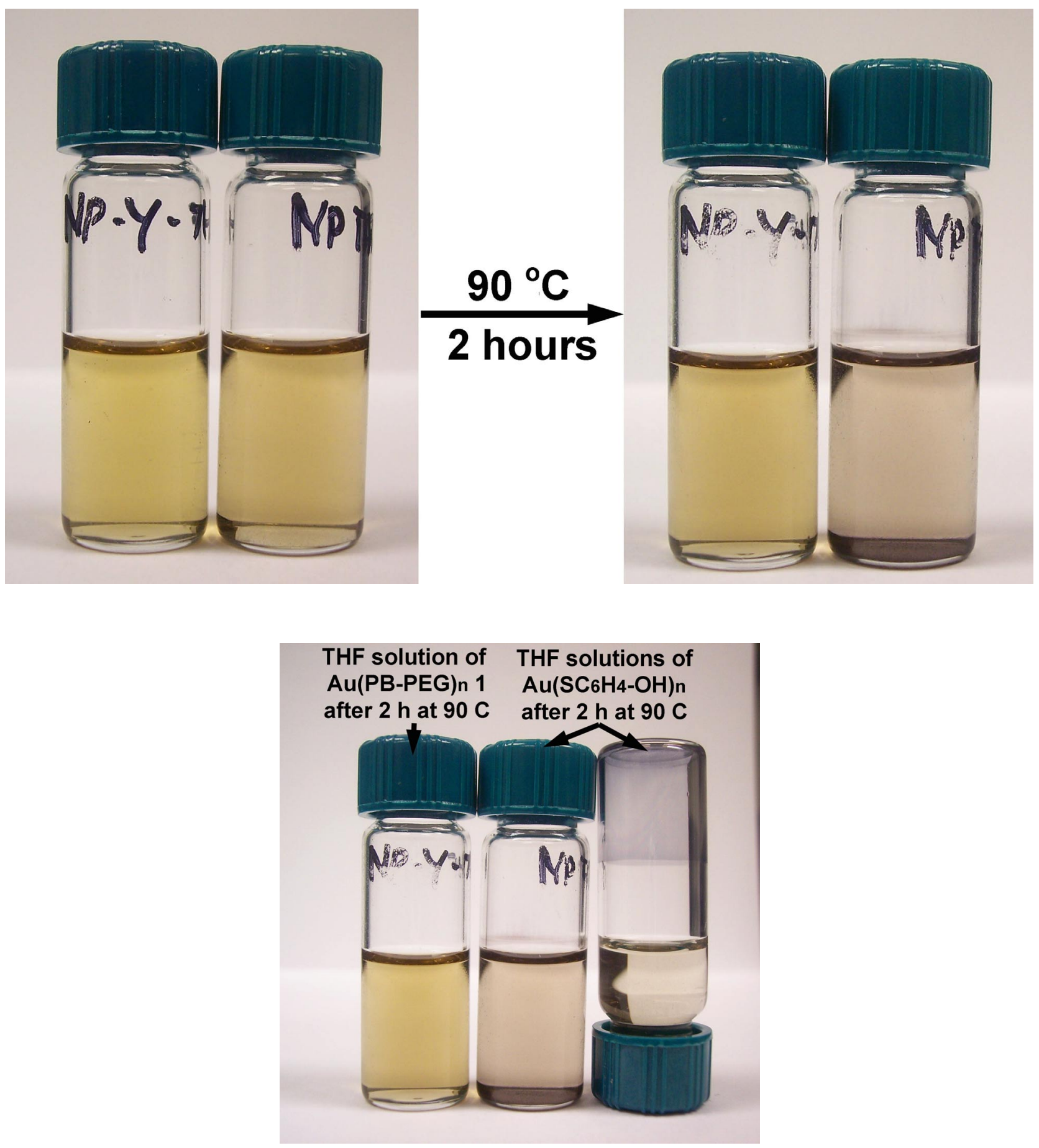

Figure S11. Top: photographs of THF solutions of $\mathrm{Au}(\mathrm{PB}-\mathrm{PEG})_{\mathrm{n}} \mathbf{1}$ (left) and mercaptophenolcoated $\mathrm{Au}\left(\mathrm{S}-\mathrm{C}_{6} \mathrm{H}_{4}-\mathrm{OH}\right)_{\mathrm{n}} \mathrm{NPs}$ before and after annealing at $90{ }^{\circ} \mathrm{C}$. The former did not change, whereas mercaptophenol-coated Au NPs were gradually changing their color from brown to red (due to Oswald ripening) until they completely agglomerated and formed black precipitate on the bottom and a thin film on the inner walls of the vial. See the bottom photograph with an inverted vial after the $2 \mathrm{~h}$ annealing. 